\title{
Effect of balanced crystalloids versus saline on urinary biomarkers of acute kidney injury in critically ill adults
}

Blake E. Funke ${ }^{1}$, Karen E. Jackson², Wesley H. Self ${ }^{3}$, Sean P. Collins ${ }^{3}$, Christina T. Saunders ${ }^{4}$, Li Wang ${ }^{4}$, Jeffrey D. Blume, ${ }^{4}$ Nancy Wickersham², Ryan M. Brown², Jonathan D. Casey², Gordon R. Bernard², Todd W. Rice², Edward D. Siew ${ }^{5+}$, Matthew W. Semler ${ }^{2^{*+}} \mathbb{D}$, for the SMART Investigators and the Pragmatic Critical Care Research Group

\begin{abstract}
Background: Recent trials have suggested use of balanced crystalloids may decrease the incidence of major adverse kidney events compared to saline in critically ill adults. The effect of crystalloid composition on biomarkers of early acute kidney injury remains unknown.

Methods: From February 15 to July 15, 2016, we conducted an ancillary study to the Isotonic Solutions and Major Adverse Renal Events Trial (SMART) comparing the effect of balanced crystalloids versus saline on urinary levels of neutrophil gelatinase-associated lipocalin (NGAL) and kidney injury molecule-1 (KIM-1) among 261 consecutivelyenrolled critically ill adults admitted from the emergency department to the medical ICU. After informed consent, we collected urine $36 \pm 12 \mathrm{~h}$ after hospital admission and measured NGAL and KIM-1 levels using commercially available ELISAs. Levels of NGAL and KIM-1 at $36 \pm 12 \mathrm{~h}$ were compared between patients assigned to balanced crystalloids versus saline using a Mann-Whitney $U$ test.
\end{abstract}

Results: The 131 patients (50.2\%) assigned to the balanced crystalloid group and the 130 patients (49.8\%) assigned to the saline group were similar at baseline. Urinary NGAL levels were significantly lower in the balanced crystalloid group (median, $39.4 \mathrm{ng} / \mathrm{mg}$ [IQR 9.9 to 133.2]) compared with the saline group (median, 64.4 ng/mg [IQR 27.6 to 339.9]) $(P<0.001)$. Urinary KIM-1 levels did not significantly differ between the balanced crystalloid group (median, $2.7 \mathrm{ng} / \mathrm{mg}$ [IQR 1.5 to 4.9$]$ ) and the saline group (median, $2.4 \mathrm{ng} / \mathrm{mg}$ [IQR 1.3 to 5.0]) $(P=0.36)$.

Conclusions: In this ancillary analysis of a clinical trial comparing balanced crystalloids to saline among critically ill adults, balanced crystalloids were associated with lower urinary concentrations of NGAL and similar urinary concentrations of KIM-1, compared with saline. These results suggest only a modest reduction in early biomarkers of acute kidney injury with use of balanced crystalloids compared with saline.

Trial registration: ClinicalTrials.gov number: NCT02444988. Date registered: May 15, 2015.

Keywords: Critical care, Resuscitation, Renal insufficiency, Dialysis, Sodium chloride, Sepsis

\footnotetext{
* Correspondence: matthew.w.semler@vumc.org

${ }^{\dagger}$ Edward D. Siew and Matthew W. Semler contributed equally to this work.

${ }^{2}$ Division of Allergy, Pulmonary, and Critical Care Medicine, Vanderbilt

University Medical Center, C-1216 MCN, 1161 21st Ave South, Nashville, TN

37232, USA

Full list of author information is available at the end of the article
}

\section{$\triangle B M C$}

C C The Author(s). 2021 Open Access This article is licensed under a Creative Commons Attribution 4.0 International License, which permits use, sharing, adaptation, distribution and reproduction in any medium or format, as long as you give appropriate credit to the original author(s) and the source, provide a link to the Creative Commons licence, and indicate if changes were made. The images or other third party material in this article are included in the article's Creative Commons licence, unless indicated otherwise in a credit line to the material. If material is not included in the article's Creative Commons licence and your intended use is not permitted by statutory regulation or exceeds the permitted use, you will need to obtain permission directly from the copyright holder. To view a copy of this licence, visit http://creativecommons.org/licenses/by/4.0/ The Creative Commons Public Domain Dedication waiver (http://creativecommons.org/publicdomain/zero/1.0/) applies to the data made available in this article, unless otherwise stated in a credit line to the data. 


\section{Background}

Administration of intravenous fluid is common in critical care [1]. Historically, $0.9 \%$ sodium chloride (saline) has been the most frequently administered fluid [2]. Balanced crystalloid solutions, such as lactated Ringer's solution and Plasma-Lyte, represent an increasingly used alternative to saline [3]. Recent data from clinical trials suggest that crystalloid composition may affect patient outcomes [4], including kidney injury, but the mechanistic effects of balanced crystalloids versus saline on the development of acute kidney injury (AKI) remain uncertain.

Pre-clinical studies have found the chloride content of intravenous fluids influences renal vasoconstriction [5], glomerular filtration rate [5], biomarkers of AKI [6], and incidence of AKI by clinical criteria and histology [7]. Randomized trials among healthy human volunteers suggest that intravenous solutions with high chloride content, such as $0.9 \%$ saline, may decrease renal artery blood velocity, renal cortical perfusion, and urine output [8-10]. A small randomized trial among patients undergoing major abdominal surgery found lower concentrations of urinary neutrophil gelatinase-associated lipocalin (NGAL), an early biomarker of renal tubular injury, with balanced crystalloids compared to saline [11]. Observational studies have reported lower rates of AKI [12] and renal replacement therapy (RRT) [13] among acutely ill adults treated with balanced crystalloids rather than saline. Two recent clinical trials found balanced crystalloid solutions decreased the composite of death, RRT, or persistent renal dysfunction during acute illness [4, 14]. Although plasma creatinine concentration did not differ between the balanced crystalloids and saline in these trials, serum creatinine is an insensitive marker for tubular injury. Whether the differences in clinical outcomes between balanced crystalloids and saline in these trials were mediated by kidney injury or by other mechanisms remains unknown.

NGAL and kidney injury molecule-1 (KIM-1) are glycoproteins produced in renal tubular epithelial cells in response to renal tubular injury. Through different mechanisms, both proteins appear to provide renal protection in response to tubular injury and both have been established as sensitive early urinary biomarkers of tubular injury [15-17]. NGAL functions as an iron-binding protein and a growth factor and is synthesized in both proximal and distal tubule cells. Its renal protective effect is thought to be due to its role in iron scavenging and delivery or its ability to induce cell growth and differentiation [15]. In the kidney, KIM-1 is expressed only in proximal tubule cells and is thought to facilitate remodeling of injured epithelia via clearance of apoptotic and necrotic cells.
To better understand the effect of crystalloid composition on the development of tubular injury, we conducted an ancillary study comparing urinary levels of the proximal and distal tubule biomarker NGAL and proximal tubule biomarker KIM-1 between critically ill adults assigned to balanced crystalloids versus saline as part of a large pragmatic trial [4]. We hypothesized that levels of these urinary biomarkers would be lower among patients assigned to balanced crystalloids compared with patients assigned to saline.

\section{Methods}

\section{Study design and oversight}

To examine the effects of balanced crystalloids versus saline on early biomarkers of acute tubular injury, we performed a pre-specified ancillary study within a large, pragmatic trial comparing balanced crystalloids to saline among critically ill adults. This study adheres to CONSORT guidelines.

The Isotonic Solutions and Major Adverse Renal Events Trial (SMART) was a cluster-randomized, cluster-crossover trial comparing balanced crystalloids to saline for intravenous fluid administration among 15,802 critically ill adults admitted to five intensive care units (ICUs) at Vanderbilt University Medical Center between June 1, 2015 and April 30, 2017 [4]. The SMART trial was approved by the Institutional Review Board at Vanderbilt University (IRB \#141349). Details of the design, analysis, and results of SMART have been published previously $[4,18]$.

The Vanderbilt University Emergency Medicine Biomarkers Study is an ongoing repository of biological samples collected from patients treated for acute illness in the Adult Emergency Department at Vanderbilt University Medical Center. This observational cohort study was approved by the Institutional Review Board at Vanderbilt University (IRB \#111510). Patients or their legally authorized representatives provided written informed consent before enrollment.

The cohort for the current study included all patients co-enrolled in SMART and the Vanderbilt University Emergency Medicine Biomarkers Study between February 15, 2016 and July 15, 2016.

\section{Patient population}

From February 15, 2016 through July 15, 2016, study personnel for the Vanderbilt University Emergency Medicine Biomarkers Study screened consecutive adults admitted from the emergency department (ED) to the medical ICU. Adult (age $\geq 18$ years) patients admitted from the ED, alive, and physically located in the medical ICU between 24 and $48 \mathrm{~h}$ after hospital admission were eligible. 


\section{Treatment assignment and intervention}

Per the SMART protocol, for each month of the trial, participating ICUs were assigned to use either balanced crystalloids (the treating clinician's choice of lactated Ringer's solution or Plasma-Lyte $\left.\mathrm{A}^{\circ}\right)$ or saline $(0.9 \%$ sodium chloride) for any intravenous isotonic crystalloid administration (Figure S1). Saline contains $154 \mathrm{mmol}$ per liter of sodium and chloride, whereas both lactated Ringer's solution and Plasma-Lyte $\mathrm{A}^{\bullet}$ contain lower concentrations of chloride, small amount of potassium, and buffering anions such as lactate, acetate, or gluconate (Table S1). Fluid administration in the ED was coordinated with the fluid assigned to the medical ICU. Patients, clinicians, and investigators were not blinded to group assignment.

\section{Collection of biospecimens}

After obtaining informed consent, study personnel collected $5 \mathrm{~mL}$ of urine from the patient or the patient's urinary catheter. Urine was collected as close to $36 \mathrm{~h}$ after hospital admission as feasible, and not less than 24 $\mathrm{h}$ or more than $48 \mathrm{~h}$ after hospital admission. The period of 24-48 h after hospital admission is referred to as "day 2 " throughout this manuscript. Study personnel then screened urine samples being held in the clinical lab at $4^{\circ}$ Celsius from the first $6 \mathrm{~h}$ of the patient's initial ED presentation. When samples were available, the study team collected $5 \mathrm{~mL}$ of urine. Samples from the first $6 \mathrm{~h}$ of the patient's initial ED presentation are referred to as "day 0" samples. All urine was centrifuged at 1000 g's for $10 \mathrm{~min}$ and frozen at $-80{ }^{\circ} \mathrm{C}$.

\section{Urinary biomarker measurement}

NGAL (also referred to as lipocalin-2 or oncogene 24p3) and KIM-1 (also referred to as hepatitis A virus cellular receptor 1 or $\mathrm{T}$-cell immunoglobulin and mucin domain 1) biomarkers were measured in duplicate in thawed urine by a commercially available Solid Phase Sandwich ELISA ( $\mathrm{R} \&$ D Systems, Minneapolis, MN). The coefficient of variation for each biomarker measurement was less than 10\% (Table S2). Background on the normal range and kinetics for NGAL and KIM-1 is provided in the Supplemental Methods.

\section{Data collection}

In addition to biomarker concentrations, study personnel blinded to study group assignment collected clinical data from the electronic health record. Data included preenrollment renal function, demographics, orders for intravenous fluids, plasma creatinine values, and clinical outcomes.

\section{Study outcomes}

The co-primary outcomes for the current study were urinary concentrations (scaled to urinary creatinine) of NGAL and KIM-1 on day 2.

Secondary biomarker outcomes included the change in NGAL and KIM-1 concentration (scaled to urinary creatinine concentration) from day 0 to day 2 among patients with urine available from day 0 . Secondary clinical outcomes included 30-day in-hospital mortality, stage II or greater acute kidney injury (AKI) according to Kidney Disease: Improving Global Outcomes (KDIGO) creatinine criteria [19], highest creatinine level before discharge or day 30, final creatinine value before discharge or day 30 , new receipt of renal-replacement therapy (RRT), the number of days alive and free from the ICU, mechanical ventilation, vasopressor use and RRT, and the proportion of patients who met one or more criteria for a major adverse kidney event within 30 days (MAKE30) - the composite of death, new receipt of RRT, or persistent renal dysfunction $[4,20]$.

\section{Statistical analysis}

The sample size for the current study was determined by the fixed number of patients co-enrolled in SMART and the Vanderbilt University Emergency Medicine Biomarkers Study between February 15, 2016 and July 15, 2016. Although a prospective sample size calculation was not performed, we estimated that the number of patients co-enrolled would be similar to the 270 patients enrolled in a recent secondary analysis of a randomized trial evaluating the effect of fluid management strategy on urinary NGAL and KIM-1 [21].

Analyses were conducted at the level of each patient's hospitalization in an intention-to-treat fashion. Continuous variables are reported as mean $\pm \mathrm{SD}$ or median and interquartile range (IQR); categorical variables are reported as frequencies and proportions.

The primary analysis compared urinary KIM-1 and NGAL levels on day 2 between the balanced crystalloid and saline groups using a Mann-Whitney $\mathrm{U}$ test.

We performed multiple secondary analyses. We compared secondary outcomes between groups using the Mann-Whitney $U$ test for continuous variables and the Chi-square test for categorical variables. To evaluate for heterogeneity of treatment effect (subgroup analysis), we fit proportional odds models with the co-primary outcomes as the dependent variables and independent variables of study group, prespecified potential effect modifiers, and the interaction between the two (see Supplemental Methods). To increase statistical power by controlling for variation and to account for any chance imbalances between study groups in this subset of trial participants, we compared the co-primary outcomes between study 
groups in proportional odds models adjusting for age, sex, race, receipt of mechanical ventilation, receipt of vasopressors, and diagnosis of sepsis.

A two-sided $P$ value $<0.05$ indicated statistical significance for the co-primary outcomes. All secondary analyses were considered hypothesis generating and no corrections for multiple testing were performed. All analyses were performed using $\mathrm{R}$ version 3.3.0 software ( $\mathrm{R}$ Foundation for Statistical Computing, Vienna, Austria).

\section{Results}

Derivation of the study cohort

A total of 261 patients admitted from the ED between February 15, 2016 and July 15, 2016 and located in the medical ICU on day 2 of hospital admission provided informed consent for biospecimen collection and were included in the current analysis (Figure S2). Of these, $131(50.2 \%)$ were assigned to the balanced crystalloid group in SMART and 130 (49.8\%) were assigned to the

Table 1 Patient Characteristics at Baseline

\begin{tabular}{|c|c|c|}
\hline Patient Characteristics $^{\mathrm{a}}$ & $\begin{array}{l}\text { Balanced } \\
(n=131)\end{array}$ & $\begin{array}{l}\text { Saline } \\
(n=130)\end{array}$ \\
\hline Age - years & $55[43-67]$ & $59[47-71]$ \\
\hline Men - no. (\%) & $61(47 \%)$ & $67(52 \%)$ \\
\hline Caucasian - no. (\%) & $112(85 \%)$ & $106(82 \%)$ \\
\hline Weight - kg & 82 [68-98] & 78 [68-95] \\
\hline \multicolumn{3}{|l|}{ Medical comorbidities - no. (\%) } \\
\hline Hypertension & $79(60 \%)$ & $69(53 \%)$ \\
\hline Diabetes & $52(40 \%)$ & $49(38 \%)$ \\
\hline Chronic respiratory failure & $40(31 \%)$ & $39(30 \%)$ \\
\hline Immunosuppression & $30(23 \%)$ & $35(27 \%)$ \\
\hline Coronary artery disease & $31(24 \%)$ & $24(18 \%)$ \\
\hline \multicolumn{3}{|l|}{ Renal comorbidities - no. (\%) } \\
\hline Chronic kidney disease, stage III or greater ${ }^{b}$ & $18(14 \%)$ & $14(11 \%)$ \\
\hline \multicolumn{3}{|c|}{ Location immediately prior to the intensive care unit - no. (\%) } \\
\hline Emergency department & $108(82 \%)$ & $105(81 \%)$ \\
\hline Operating room & $2(2 \%)$ & $2(2 \%)$ \\
\hline Hospital ward & $21(16 \%)$ & $23(18 \%)$ \\
\hline \multicolumn{3}{|l|}{ Admitting diagnosis - no. (\%) } \\
\hline Sepsis or septic shock & $36(27 \%)$ & $33(25 \%)$ \\
\hline Respiratory failure & $28(21 \%)$ & $34(26 \%)$ \\
\hline Gastrointestinal bleed & $16(12 \%)$ & $19(15 \%)$ \\
\hline Mechanical ventilation - no. (\%) & $17(13 \%)$ & $19(15 \%)$ \\
\hline Vasopressors - no. (\%) & $10(8 \%)$ & $12(9 \%)$ \\
\hline APACHE II score & $11[7.5-15]$ & $13[9-16.2]$ \\
\hline Baseline creatinine ${ }^{c}-\mathrm{mg} / \mathrm{dL}$ & $0.77[0.67-0.90]$ & $0.78[0.65-0.90]$ \\
\hline Day 0 NGAL level ${ }^{e}$ & 70 [33-442] & $81[28-461]$ \\
\hline Day 0 KIM-1 level & $3.5[2.1-6.9]$ & $3.1[1.4-5.5]$ \\
\hline Acute kidney injury, stage $\|$ or greater ${ }^{d}$ & $35(26 \%)$ & $34(27 \%)$ \\
\hline
\end{tabular}

${ }^{a}$ Continuous data are presented as median [25th percentile - 75th percentile] unless otherwise noted. Categorical data are presented as number (no.) and percentage (\%). There were no significant differences in baseline characteristics between the two study groups ( $P$ values range from 0.05 to 0.78$)$

${ }^{b}$ Chronic kidney disease stage III or greater is defined as a glomerular filtration rate less than $60 \mathrm{ml} / \mathrm{min}$ per $1.73 \mathrm{~m}{ }^{2}$ as calculated by the Chronic Kidney Disease Epidemiology (CKD-EPI) Collaboration equation [22] using the patient's baseline creatinine value

'Baseline creatinine for the study is defined as the lowest plasma creatinine measured in the 12 months prior to hospitalization if available, otherwise the lowest plasma creatinine measured between hospitalization and intensive care unit admission; using an estimated creatinine only for patients without an available plasma creatinine between 12 months prior to hospitalization and the time of ED presentation. A total of 37 patients (28\%) in the balanced crystalloid group and 45 patients (35\%) in the saline group did not have a measured plasma creatinine value available between 12 months prior to hospital admission and the time of intensive care unit admission

${ }^{\mathrm{d}}$ Acute kidney injury, stage II or greater is defined according to Kidney Disease Improving Global Outcomes (KDIGO) creatinine criteria [19] as a first plasma creatinine value at time of ED presentation at least $200 \%$ of the baseline value OR both (1) greater than $4.0 \mathrm{mg} / \mathrm{dL}$ and (2) increased at least $0.3 \mathrm{mg} / \mathrm{dL}$ from the baseline value

${ }^{\mathrm{e}}$ Day 0 urine samples were available for 60 patients (46\%) in the balanced group and 51 patients (39\%) in the saline group 
saline group. A total of 111 of the 261 patients (42.5\%) also had urine samples available from day 0 , of whom 60 (54.1\%) were in the balanced crystalloid group and 51 (45.9\%) were in the saline group.

\section{Baseline characteristics}

Among the 261 patients enrolled, median age was 58 years, $12 \%$ of patients had stage III or greater chronic kidney disease (CKD) (as defined in the supplemental methods), 26\% had stage II or greater AKI at ED presentation (prior to the intervention), $26 \%$ had a diagnosis of sepsis, and $8 \%$ were receiving vasopressors. Patients assigned to the balanced crystalloid group $(n=131)$ and saline group $(n=130)$ were similar at baseline with respect to age, gender, pre-existing chronic kidney disease, and baseline plasma creatinine concentration (Table 1, Tables S3-4). Among the 111 patients with urinary biomarkers measured on day 0 , the balanced crystalloid and saline groups did not differ with regard to urinary NGAL level (median, 70 [IQR, 33 to 442] vs 81 [IQR, 28 to 461]; $P=0.89$ ) or KIM-1 level (median, 3.5 [IQR, 2.1 to 6.9 ] vs 3.1 [IQR, 1.4 to 5.5 ]; $P=0.36$ ).

\section{Fluid therapy and electrolytes}

In the ED prior to ICU admission, patients in the balanced crystalloid group received a mean of $991 \pm$ $1189 \mathrm{~mL}$ of balanced crystalloids and $307 \pm 787 \mathrm{~mL}$ of saline, whereas patients in the saline group received a mean of $13 \pm 98 \mathrm{~mL}$ of balanced crystalloids and
$1372 \pm 1654 \mathrm{~mL}$ of saline $(P<0.001) \quad$ (Table S5). Between ICU admission and day 2, patients in the balanced crystalloid group received a mean of $1911 \pm$ $2277 \mathrm{~mL}$ of balanced crystalloid and $153 \pm 618 \mathrm{~mL}$ of saline, whereas patients in the saline group received a mean of $256 \pm 1499$ of balanced crystalloid and $1641 \pm 1973 \mathrm{~mL}$ of saline $(P<0.001)$. The volume of non-study intravenous fluids did not differ between groups (Table S6). Plasma chloride concentrations were lower and plasma bicarbonate concentrations were higher in the balanced crystalloid group compared with the saline group (Table S7).

\section{Co-primary outcomes}

Among all 261 patients, urinary NGAL levels on day 2 were significantly lower in the balanced crystalloid group (median, 39.4 ng/mg; IQR, 9.9 to 133.2) compared with the saline group (median, $64.4 \mathrm{ng} / \mathrm{mg}$; IQR 27.6 to 339.9 ) $(P<0.001)$. Urinary KIM-1 levels on day 2 did not differ between the balanced crystalloid group (median, $2.7 \mathrm{ng} /$ mg; IQR, 1.5 to 4.9 ) and the saline group (median, 2.4 ng/mg; IQR, 1.3 to 5.0$)(P=0.36)$ (Fig. 1). Results were similar in analyses adjusting for pre-specified baseline covariates (Tables S8-9).

In subgroup analyses, the effect of balanced crystalloids versus saline on urinary NGAL levels on day 2 appeared to be greater among patients without sepsis, patients with higher APACHE II scores, and patients with AKI on ED presentation (Fig. 2).

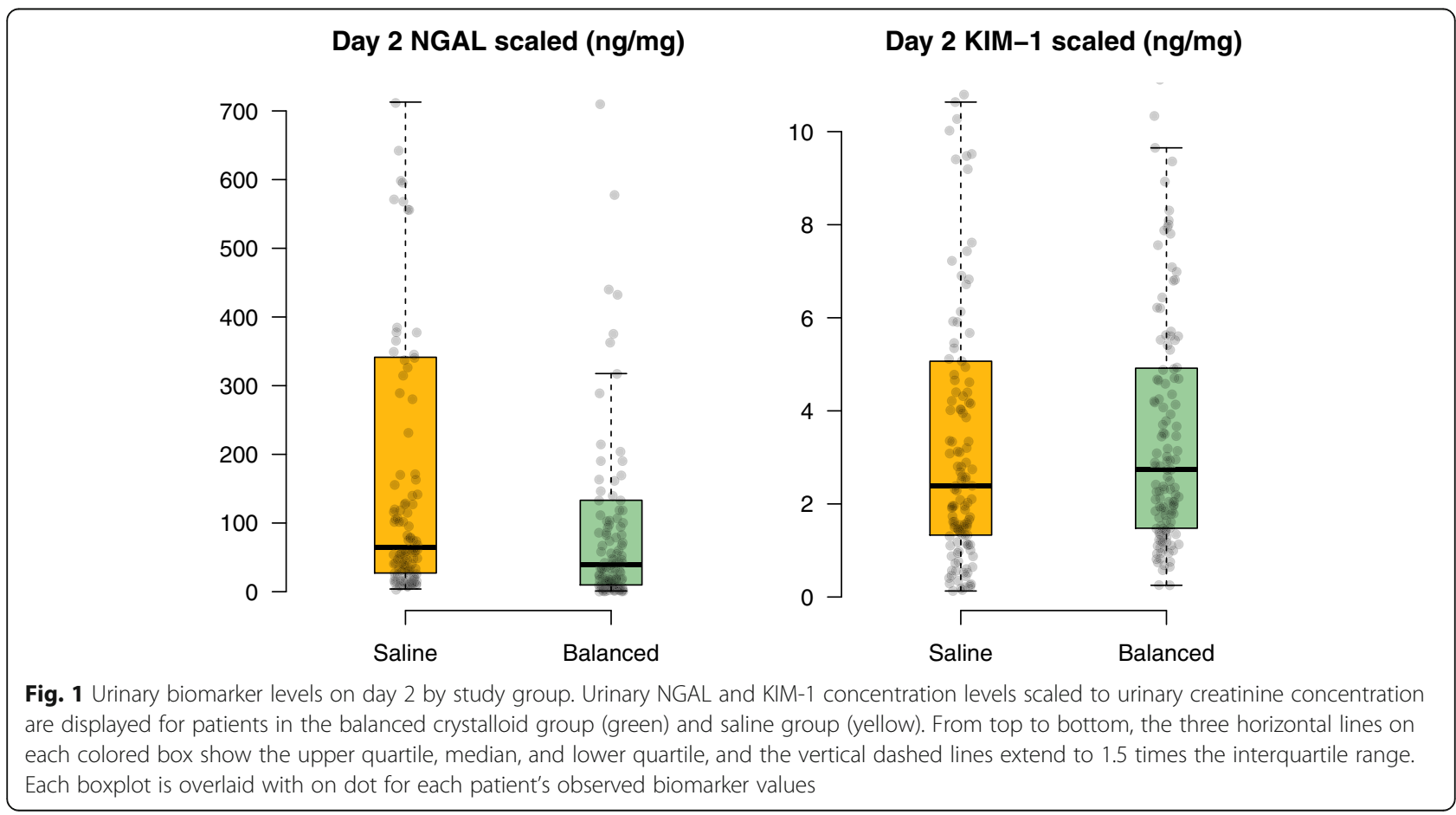




\section{Secondary outcomes}

There was no significant difference between groups in secondary clinical outcomes, including stage II or greater AKI, 30-day in-hospital mortality, new receipt of renal replacement therapy, and major adverse kidney events within 30 days (Table 2 and Tables S10-11).

Among the 111 patients with urine available from ED presentation, urinary NGAL levels on day 2 appeared to be lower in the balanced crystalloid group (median, $47.4 \mathrm{ng} / \mathrm{mg} ; \mathrm{IQR}, 8.4$ to 150.6 ) compared with the saline group (median, 59.4 $\mathrm{ng} / \mathrm{mg}$; IQR, 27.9 to 301.7) $(P=0.059)$. Urinary KIM-1 levels on day 2 did not differ between the balanced crystalloid group (median, $2.6 \mathrm{ng} / \mathrm{mg}$; IQR, 1.5 to 5.8 ) and the saline group (median, $2.6 \mathrm{ng} / \mathrm{mg}$; IQR, 1.5 to 5.0$)(P=0.78)$. The change in urinary NGAL level from day 0 to day 2 was $-27.7 \mathrm{ng} / \mathrm{mg}$ (IQR -293.4 to 2.3 ) in the balanced crystalloid group versus $-7.8 \mathrm{ng} / \mathrm{mg}$ (IQR 115.9 to 25.7$)$ in the saline group $(P=0.14)$. Change in urinary KIM-1 level from day 0 to day 2 was $0.59 \mathrm{ng} / \mathrm{mg}$ (IQR -2.9 to 1.21 ) in the balanced crystalloid group versus $-0.29 \mathrm{ng} / \mathrm{mg}$ (IQR -1.48 to 0.48 ) in the saline group $(P=0.79)$ (Fig. 3, Figure S3).

\section{Discussion}

In this ancillary analysis of a large clinical trial comparing balanced crystalloids to saline among critically ill adults, balanced crystalloids were associated with modestly lower urinary concentrations of NGAL and similar urinary concentrations of KIM-1 compared with saline. These findings provide the first examination of the effect of crystalloid composition on biomarkers of early AKI in critical illness and inform one of the mechanisms by which crystalloid composition may affect clinical outcomes.

Prior studies have evaluated the effect of balanced crystalloids versus saline on biomarkers of AKI in animal models. Zhou et al. reported that in a rat model of sepsis, resuscitation with a balanced crystalloid solution resulted in lower urinary concentrations of NGAL compared to saline [6]. In this model, the higher NGAL concentrations with saline corresponded with higher stages of AKI and loss of brush border, vacuolization, and dilation of the tubular lumen on renal histology.

Recent studies have examined the effect of crystalloid composition on biomarkers of AKI in humans. In a randomized controlled trial among adult patients undergoing

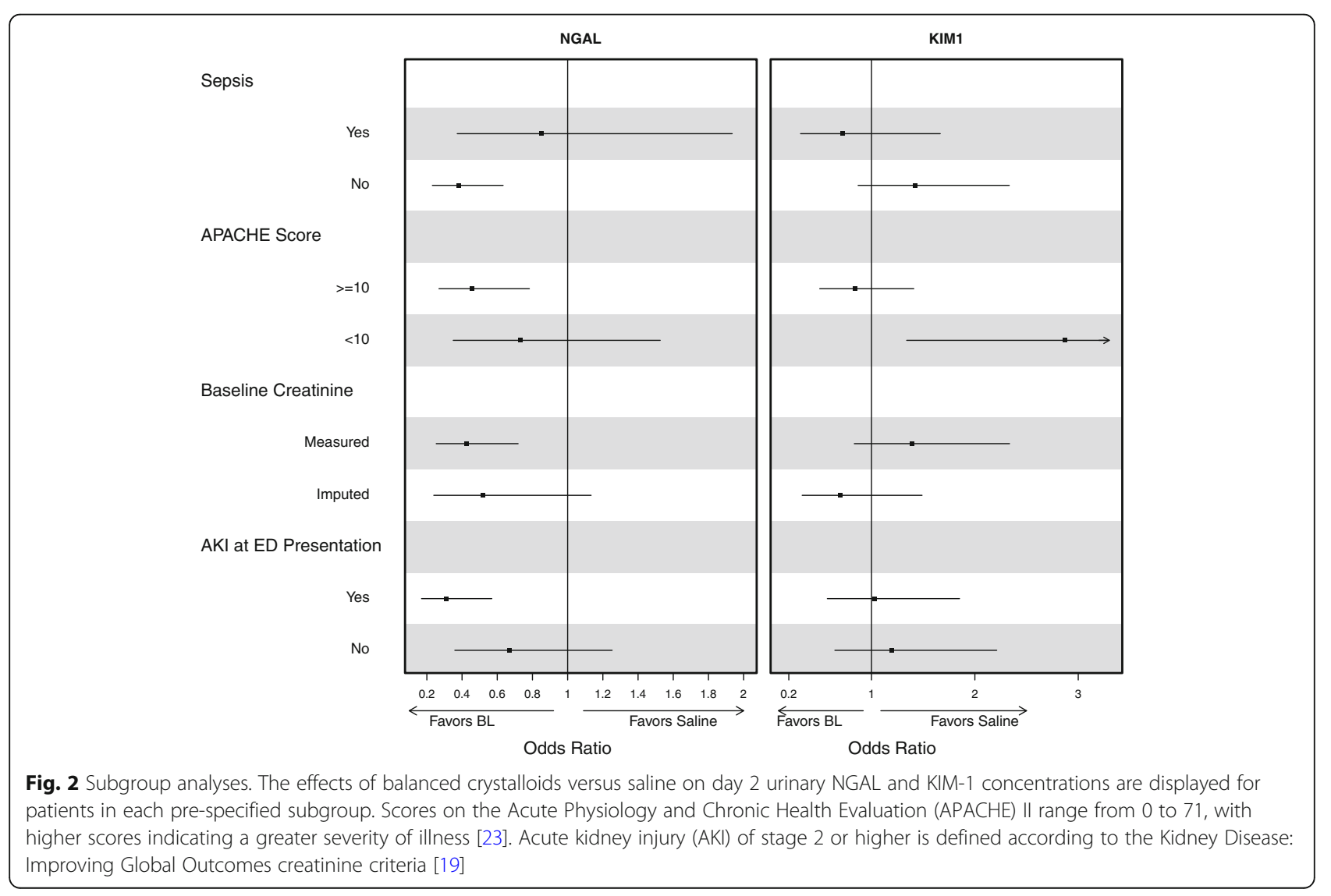


Table 2 Clinical Outcomes

\begin{tabular}{|c|c|c|c|c|}
\hline Outcome $^{a}$ & $\mathrm{n}$ & Balanced $(n=131)$ & Saline $(n=130)$ & $P$ Value \\
\hline \multicolumn{5}{|l|}{ Primary Outcomes } \\
\hline Day 2 urinary NGAL level - ng/mg (IQR) & 261 & $39.4[9.9-133.2]$ & $64.4[27.6-339.9]$ & $<0.001$ \\
\hline Day 2 urinary KIM-1 level - ng/mg (IQR) & 261 & $2.7[1.5-4.9]$ & $2.4[1.3-5.0]$ & 0.36 \\
\hline \multicolumn{5}{|l|}{ Secondary Biomarker Outcomes } \\
\hline Change in NGAL level from day 0 to 2 & 111 & $-27.7[-293.4-2.3]$ & $-7.8[-115.9-25.7]$ & 0.14 \\
\hline Change in KIM-1 level from day 0 to 2 & 111 & $-0.59[-2.9-1.21]$ & $-0.29[-1.48-0.48]$ & 0.79 \\
\hline \multicolumn{5}{|l|}{ Secondary Clinical Outcomes } \\
\hline 30-day in-hospital mortality - no. (\%) & 261 & $5(4 \%)$ & $10(8 \%)$ & - \\
\hline Major Adverse Kidney Event within 30 days - no. $(\%)^{\mathrm{b}}$ & 261 & $13(10 \%)$ & $17(13 \%)$ & - \\
\hline Intensive care unit-free days ${ }^{c}$ & 260 & $25.0[23.0-26.0]$ & $25.0[23.0-25.0]$ & - \\
\hline Mean \pm SD & & $22.5 \pm 6.5$ & $21.8 \pm 7.2$ & \\
\hline$V^{V}$ tilator-free days ${ }^{c}$ & 260 & $28.0[28.0-28.0]$ & $28.0[27.0-28.0]$ & - \\
\hline Mean \pm SD & & $25.8 \pm 6.3$ & $25.1 \pm 7.6$ & \\
\hline Vasopressor-free days $^{c}$ & 260 & $28.0[27.0-28.0]$ & $28.0[28.0-28.0]$ & - \\
\hline Mean \pm SD & & $26.0 \pm 6.2$ & $25.6 \pm 7.1$ & \\
\hline Renal replacement therapy-free days ${ }^{c}$ & 260 & $28.0[28.0-28.0]$ & $28.0[28.0-28.0]$ & - \\
\hline Mean \pm SD & & $26.6 \pm 5.8$ & $25.3 \pm 7.9$ & \\
\hline Stage II or greater AKI developing after enrollment - no. $(\%)^{d}$ & 261 & $50(39 \%)$ & $48(37 \%)$ & - \\
\hline New receipt of RRT & 261 & $1(1 \%)$ & $3(2 \%)$ & - \\
\hline \multicolumn{5}{|l|}{ Creatinine, mg/dL } \\
\hline Highest before discharge or day 30 & 260 & $1.32[0.95-1.96]$ & $1.17[0.81-1.85]$ & - \\
\hline Final value before discharge or 30 days & 259 & $0.84[0.72-1.15]$ & $0.79[0.68-1.07]$ & - \\
\hline
\end{tabular}

${ }^{a}$ Continuous data are presented as median [25th percentile - 75th percentile] unless otherwise noted. Categorical data are presented as number (no.) and percentage (\%)

${ }^{b}$ Major Adverse Kidney Events within 30 days (MAKE30) is the composite of death, receipt of new renal replacement therapy, or final creatinine $\geq 200 \%$ baseline, all censored at the first of hospital discharge or 30 days after intensive care unit admission

'Intensive care unit-, ventilator-, vasopressor-, and renal replacement therapy-free days refer to the number of days alive and free from the specified therapy in the first 28 days after enrollment

${ }^{\mathrm{d}}$ Stage II or greater acute kidney injury (AKI) developing after enrollment is defined using the Kidney Disease Improving Global Outcomes (KDIGO) creatinine criteria [19] as (1) any creatinine value between enrollment and discharge or 30 days that is (a) increased at least $0.3 \mathrm{mg} / \mathrm{dL}$ from a preceding post-enrollment value and (b) at least $200 \%$ of the baseline value, at least $200 \%$ of a preceding post-enrollment value, or at least $4.0 \mathrm{mg} / \mathrm{dL}$; ( 2 ) urine output $<0.5 \mathrm{ml} / \mathrm{k} / \mathrm{h}$ for at least 12 consecutive hours; or (3) new receipt of renal replacement therapy

major abdominal surgery, Volta et al. found that intraoperative use of balanced solutions rather than unbalanced solutions resulted in lower urinary NGAL concentrations at the end of surgery, $2 \mathrm{~h}$ after surgery, and on postoperative day 1 [11]. Additionally, Dey et al. reported that in patients undergoing elective craniotomy for supratentorial brain tumors, serum NGAL concentrations were significantly lower $3 \mathrm{~h}$ after surgery in patients randomized to intra-operative Plasma-Lyte compared to saline [24].

Our findings are consistent with this prior research suggesting that crystalloid composition may influence urinary concentrations of NGAL. High chloride delivery to the distal tubule has been hypothesized to increase tubuloglomerular feedback, decrease renal blood flow, and contribute to ischemic injury to the kidney [5]. These effects might be greater among patients who have already experienced acute kidney injury and might influence recovery from kidney injury instead of or in addition to development of new kidney injury. In the current study, the difference between balanced crystalloids and saline in urinary NGAL concentration was larger among patients with AKI at the time of presentation. This is similar to the finding in prior trials that the effect of balanced crystalloids versus saline on clinical outcomes appeared to be greatest among patients with AKI at the time of presentation [14].

To our knowledge, this is also the first study to compare the effect of balanced crystalloids versus saline crystalloids on urinary KIM-1 levels. Prior work has examined the effect of hypertonic saline on urinary KIM-1. In a study by Mose et al. [25], healthy subjects were given an intravenous load of $3 \%$ saline over $60 \mathrm{~min}$ accompanied by placebo or furosemide. In subjects randomized to 3\% saline and placebo, urinary KIM-1 levels were significantly increased from baseline in the 150210 min after infusion. 
Delta scaled NGAL

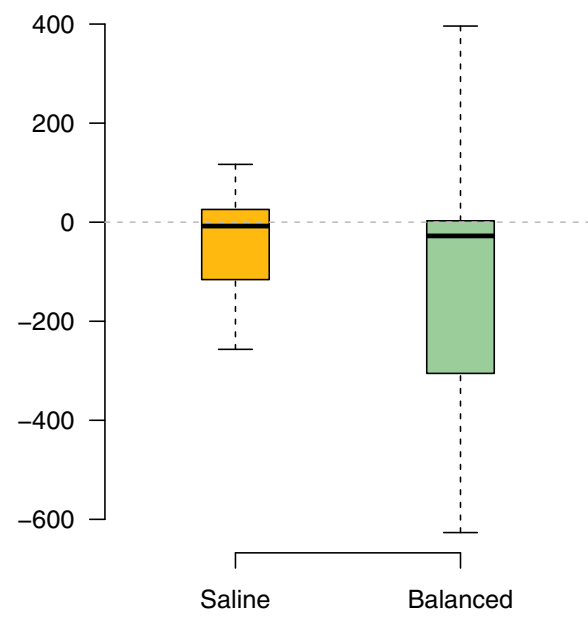

Delta scaled KIM 1

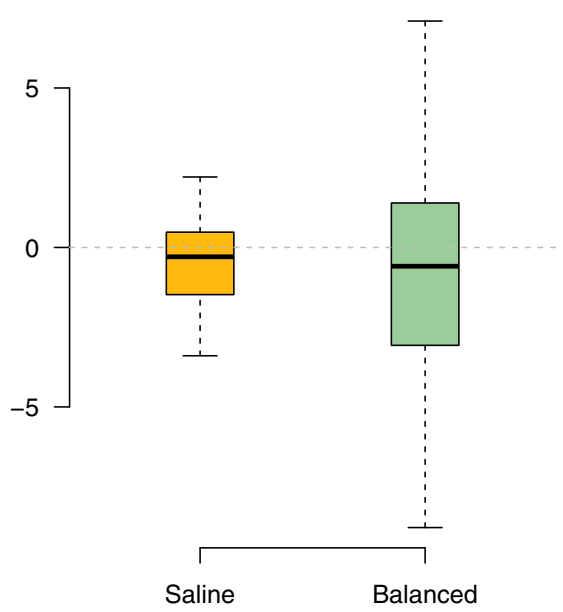

Fig. 3 Change in urinary biomarker levels from day 0 to day 2 by study group. The change in urinary NGAL and KIM-1 concentration from emergency department presentation to day 2 of hospital admission scaled to urinary creatinine concentration is displayed for patients in the balanced crystalloid group (green) and saline group (yellow), among the 111 patients with urine available at both time points. From top to bottom, the three horizontal lines on each colored box show the upper quartile, median, and lower quartile, and the vertical dashed lines extend to 1.5 times the interquartile range

Several potential reasons might explain why we observed differences between balanced crystalloids and saline in urinary NGAL levels but not KIM-1 levels at $36 \mathrm{~h}$. First, differences in the kinetics of expression for the two biomarkers may have contributed to these findings. For example, in patients in which the timing of the kidney insult is known (e.g. cardiac surgery), urinary NGAL levels appear to peak sooner (hours) in patients with AKI compared to similar studies with KIM-1, which tended to peak at 3 days after surgery [26, 27]. Thus, it is possible that any putative effect of crystalloid type on kidney injury biomarker expression may not have yet been fully manifest with urinary KIM-1 at the timepoint we assessed. Differences may also exist in the threshold expression of these biomarkers in response to crystalloid. For example, overall levels of expression for each marker were generally modest compared to other studies in patients with established acute tubular necrosis $[16,28,29]$. It is known that mRNA expression of NGAL in response to ischemia is produced in the distal tubule, with much of the proximal tubular protein potentially taken up post-filtration. Thus, it is possible that the modest amounts of saline delivered may not have been enough to drive differences in severe proximal tubular injury driving KIM-1 expression or loss of NGAL, but potentially were sufficient to induce expression of NGAL in the distal tubule. Alternatively, the effects of crystalloid composition may be resulting in differences in non-renal expression of NGAL. NGAL production is not limited to the kidney and is produced in multiple cell types during systemic inflammation.
Thus, it is possible that differences in urinary level of NGAL may represent differences in systemic production of NGAL in response to saline administration, which may be more evident because of the overall low levels of each biomarker.

The clinical significance of our findings is uncertain. The normal urinary concentration of NGAL is around $20 \mathrm{ng} / \mathrm{m} \mathrm{[30]} \mathrm{and} \mathrm{prior} \mathrm{work} \mathrm{has} \mathrm{suggested} \mathrm{cut-off} \mathrm{value}$ of $>150 \mathrm{ng} / \mathrm{ml}$ as diagnostic of AKI [29]. In this study, though there was a statistically significant difference in urinary concentrations of NGAL between groups, the absolute value was small and may not represent clinically relevant tubular injury.

In the original SMART trial, differences in the composite MAKE30 outcome appeared to be driven primarily by differences in renal replacement therapy and death rather than by changes in creatinine. The low overall levels of urinary biomarkers and only modest difference in NGAL between groups in the current study may support the hypothesis that differences in clinical outcomes in SMART were mediated by mechanisms other than direct tubular injury (e.g. acidosis, systemic inflammation, hemodynamic instability). Use of balanced crystalloids appeared to cause lower vasopressor requirements in both a recent randomized trial during major abdominal surgery and a secondary analysis of patients with sepsis in the SMART trial $[31,32]$.

Our study has several strengths. The crystalloid solution patients received was determined by study group assignment, generating similar groups and minimizing indication 
bias. Beginning delivery of the assigned crystalloid during fluid resuscitation in the ED ensured that patients did not receive significant volumes of the non-assigned crystalloid prior to enrollment and captured the period of highest risk for acute kidney injury [21]. Urinary concentration of NGAL and KIM-1 are accepted biomarkers of early AKI and were measured using commercially available ELISAs facilitating reproducibility.

This study also has several limitations. In a subgroup analysis of a clinical trial, chance imbalances in patient characteristics at baseline, such as urinary biomarker level, might explain differences in observed outcomes. Many patients did not have urine available from the time of presentation to the ED, limiting the analyses attempting to adjust for baseline biomarker concentration. The small sample size precluded sufficient statistical power to detect small differences in clinical outcomes. Patients were enrolled from a single medical ICU, limiting generalizability. Treating clinicians were aware of the composition of the assigned crystalloid. The average volume of intravenous crystalloid administered was relatively small. Long-term followup of kidney function beyond hospital discharge was unavailable.

\section{Conclusions}

In this ancillary analysis of a clinical trial comparing balanced crystalloids to saline among critically ill adults, balanced crystalloids were associated with modestly lower urinary concentrations of NGAL and similar urinary concentrations of KIM-1, compared with saline. Future research should examine mechanisms other than tubular injury by which use of balanced crystalloids rather than saline might affect receipt of renal replacement therapy and death.

\section{Supplementary Information}

The online version contains supplementary material available at https://doi. org/10.1186/s12882-021-02236-x.

\footnotetext{
Additional file 1: Supplemental Methods. Table S1. Composition of the study fluids. Table S2. Coefficient of variation for urinary biomarkers. Table S3. Elixhauser comorbidity index. Table S4. Baseline laboratory values. Table S5. Volume of intravenous isotonic crystalloid by study group. Table S6. Volume of non-study intravenous crystalloid. Table S7. Laboratory values. Table S8. Multivariable model for urinary NGAL concentration. Table S9. Multivariable model for urinary KIM-1 concentration. Table S10. Multivariable model for Major Adverse Kidney Events within 30 days. Table S11. Highest stage of acute kidney injury developing after enrollment. Figure S1. Study group assignment during the trial. Figure S2. Flow of participants through the trial. Figure S3. Urinary biomarker levels at ED presentation and $36 \mathrm{~h}$. The median (horizontal bar), interquartile range (colored box), 95\% confidence interval (dashed line) for urinary NGAL and KIM-1 concentration at time of emergency department presentation (Day 0, 111 patients) and $36 \mathrm{~h}$ after hospital admission (Day 2, 261 patients) scaled to urinary creatinine concentration are displayed for patients in the balanced crystalloid group and saline group
}

\section{Abbreviations}

SMART: Isotonic Solutions and Major Adverse Renal Events Trial;

NGAL: Neutrophil gelatinase-associated lipocalin; KIM-1: Kidñey injury molecule-1; AKI: Acute kidney injury; RRT: Renal replacement therapy; ICUs: Intensive care units; ED: Emergency department; KDIGO: Kidney Disease: Improving Global Outcomes; MAKE30: Major adverse kidney event within 30 days; IQR: Interquartile range; CKD: Chronic kidney disease; APAC HE: Acute Physiology and Chronic Health Evaluation; CKD-EPI: Chronic Kidney Disease Epidemiology

\section{Acknowledgements}

Not applicable

\section{Authors' contributions}

Study concept and design: W.H.S., T.W.R., E.D.S., M.W.S. Acquisition of data: B.E.F., K.E.J., W.H.S., S.P.C., N.W., R.M.B., T.W.R., E.D.S., M.W.S. Analysis and interpretation of data: B.E.F., K.E.J., W.H.S., S.P.C., C.T.S., L.W., J.D.B., N.W., R.M.B., J.D.C., G.R.B., T.W.R., E.D.S., M.W.S. Drafting of the manuscript: B.E.F., E.D.S., M.W.S. Critical revision of the manuscript for important intellectual content: B.E.F., K.E.J., W.H.S., S.P.C., C.T.S., L.W., J.D.B., N.W., R.M.B., J.D.C., G.R.B., T.W.R., E.D.S., M.W.S. Statistical analysis: C.T.S., L.W., J.D.B. Study supervision: W.H.S., G.R.B., T.W.R., E.D.S., M.W.S. C.T.S., L.W., J.D.B. had full access to all the data in the study and take responsibility for the integrity of the data and the accuracy of the data analysis. The author(s) read and approved the final manuscript

\section{Funding}

Financial support for the study was provided by the Vanderbilt Institute for Clinical and Translational Research (UL1 TR000445 and UL1TR002243 from NCATS/NIH). T.W.R. was supported in part by NHLBI (U01 HL123033). E.D.S. was supported in part by the Vanderbilt O'Brien Kidney Center (5P30 DK114809-02) and Vanderbilt Nephrology Divisional Funds. M.W.S. was supported in part by the NHLBI (K23HL143053). The funding institutions had no role in (1) conception, design, or conduct of the study, (2) collection, management, analysis, interpretation, or presentation of the data, (3) preparation, review, or approval of the manuscript, or (4) the decision to submit for publication.

\section{Availability of data and materials}

The datasets used and/or analyzed during the current study are available from the corresponding author on reasonable request.

\section{Ethics approval and consent to participate}

This study was approved by the Institutional Review Board at Vanderbilt University (IRB \#111510). Patients or their legally authorized representatives provided written informed consent before enrollment.

\section{Consent for publication}

Not applicable.

\section{Competing interests}

W.H.S. reported personal fees from Baxter during the conduct of this study T.W.R. reported personal fees from Cumberland Pharmaceuticals, Inc. and Avisa Pharma, LLC outside the submitted work. The remaining authors declare that they have no competing interests.

\section{Author details}

${ }^{1}$ Department of Medicine, Vanderbilt University Medical Center, Nashville, TN, USA. ${ }^{2}$ Division of Allergy, Pulmonary, and Critical Care Medicine, Vanderbilt University Medical Center, C-1216 MCN, 1161 21st Ave South, Nashville, TN 37232, USA. ${ }^{3}$ Department of Emergency Medicine, Vanderbilt University Medical Center, Nashville, TN, USA. ${ }^{4}$ Department of Biostatistics, Vanderbilt University Medical Center, Nashville, TN, USA. ${ }^{5}$ Division of Nephrology and Hypertension, Vanderbilt Center for Kidney Disease (VCKD) and Integrated Program for AKI (VIP-AKI), Vanderbilt University Medical Center, Nashville, TN, USA. 
Received: 4 September 2020 Accepted: 7 January 2021

Published online: 05 February 2021

\section{References}

1. Myburgh JA, Mythen MG. Resuscitation fluids. N Engl J Med. 2013;369:124351.

2. Finfer S, Liu B, Taylor C, et al. Resuscitation fluid use in critically ill adults: an international cross-sectional study in 391 intensive care units. Crit Care Lond Engl. 2010;14:R185

3. Hammond NE, Taylor C, Finfer S, et al. Patterns of intravenous fluid resuscitation use in adult intensive care patients between 2007 and 2014: An international cross-sectional study. PLoS One. 2017;12:e0176292.

4. Semler MW, Self WH, Wanderer JP, et al. Balanced crystalloids versus saline in critically ill adults. N Engl J Med. 2018;378:829-39.

5. Wilcox CS. Regulation of renal blood flow by plasma chloride. J Clin Invest 1983;71:726-35.

6. Zhou F, Peng Z-Y, Bishop JV, et al. Effects of fluid resuscitation with $0.9 \%$ saline versus a balanced electrolyte solution on acute kidney injury in a rat model of sepsis*. Crit Care Med. 2014;42:e270-8.

7. Bellomo R, Ronco C, Kellum JA, et al. Acute renal failure - definition, outcome measures, animal models, fluid therapy and information technology needs: the second international consensus conference of the acute Dialysis quality initiative (ADQI) group. Crit Care Lond Engl. 2004;8: R204-12.

8. Chowdhury AH, Cox EF, Francis ST, et al. A randomized, controlled, doubleblind crossover study on the effects of $2-L$ infusions of $0.9 \%$ saline and plasma-lyte 148 on renal blood flow velocity and renal cortical tissue perfusion in healthy volunteers. Ann Surg. 2012;256:18-24.

9. Chowdhury AH, Cox EF, Francis ST, et al. A randomized, controlled, doubleblind crossover study on the effects of 1-L infusions of $6 \%$ hydroxyethyl starch suspended in $0.9 \%$ saline (voluven) and a balanced solution (plasma volume Redibag) on blood volume, renal blood flow velocity, and renal cortical tissue perfusion in healthy volunteers. Ann Surg. 2014;259:881-7.

10. Williams EL, Hildebrand KL, McCormick SA, et al. The effect of intravenous lactated Ringer's solution versus 0.9\% sodium chloride solution on serum osmolality in human volunteers. Anesth Analg. 1999;88:999-1003.

11. Volta CA, Trentini A, Farabegoli L, et al. Effects of two different strategies of fluid administration on inflammatory mediators, plasma electrolytes and acid/base disorders in patients undergoing major abdominal surgery: a randomized double blind study. J Inflamm. 2013;10:29.

12. Weinberg L, Li M, Churilov L, et al. Associations of fluid amount, type, and balance and acute kidney injury in patients undergoing major surgery. Anaesth Intensive Care. 2018:46:79-87.

13. Shaw AD, Bagshaw SM, Goldstein SL, et al. Major complications, mortality, and resource utilization after open abdominal surgery: 0.9\% saline compared to plasma-Lyte. Ann Surg. 2012;255:821-9.

14. Self WH, Semler MW, Wanderer JP, et al. Balanced crystalloids versus saline in noncritically ill adults. N Engl J Med. 2018;378:819-28.

15. Schmidt-Ott KM, Mori K, Li JY, et al. Dual action of neutrophil gelatinaseassociated lipocalin. J Am Soc Nephrol JASN. 2007;18:407-13.

16. Han WK, Bailly V, Abichandani R, et al. Kidney injury Molecule-1 (KIM-1): a novel biomarker for human renal proximal tubule injury. Kidney Int. 2002;62: 237-44

17. Bonventre JV. Kidney injury molecule-1 (KIM-1): a urinary biomarker and much more. Nephrol Dial Transplant Off Publ Eur Dial Transpl Assoc - Eur Ren Assoc. 2009:24:3265-8.

18. Semler MW, Self WH, Wang $L$, et al. Balanced crystalloids versus saline in the intensive care unit: study protocol for a cluster-randomized, multiplecrossover trial. Trials. 2017;18:129.

19. Kidney Disease: Improving Global Outcomes (KDIGO) Acute Kidney Injury Work Group. KDIGO Clinical Practice Guideline for Acute Kidney Injury. Kidney Int. 2012;2(Suppl):8.

20. Palevsky PM, Molitoris BA, Okusa MD, et al. Design of clinical trials in acute kidney injury: report from an NIDDK workshop on trial methodology. Clin J Am Soc Nephrol CJASN. 2012;7:844-50.

21. Kellum JA, Chawla LS, Keener $C$, et al. The effects of alternative resuscitation strategies on acute kidney injury in patients with septic shock. Am J Respir Crit Care Med. 2016;193:281-7.

22. Levey AS, Stevens LA, Schmid $\mathrm{CH}$, et al. A new equation to estimate glomerular filtration rate. Ann Intern Med. 2009;150:604-12.
23. Knaus WA, Draper EA, Wagner DP, et al. APACHE II: a severity of disease classification system. Crit Care Med. 1985;13:818-29.

24. Dey A, Adinarayanan S, Bidkar PU, et al. Comparison of normal saline and balanced crystalloid (plasmalyte) in patients undergoing elective craniotomy for supratentorial brain tumors: a randomized controlled trial. Neurol India. 2018;66:1338-44.

25. Mose FH, Jörgensen AN, Vrist MH, et al. Effect of $3 \%$ saline and furosemide on biomarkers of kidney injury and renal tubular function and GFR in healthy subjects - a randomized controlled trial. BMC Nephrol. 2019;20:200.

26. Parikh CR, Coca SG, Thiessen-Philbrook H, et al. Postoperative biomarkers predict acute kidney injury and poor outcomes after adult cardiac surgery. J Am Soc Nephrol JASN. 2011;22:1748-57.

27. Parikh $\mathrm{CR}$, Thiessen-Philbrook H, Garg AX, et al. Performance of kidney injury molecule-1 and liver fatty acid-binding protein and combined biomarkers of AKI after cardiac surgery. Clin J Am Soc Nephrol CJASN. 2013:8:1079-88.

28. Vaidya VS, Waikar SS, Ferguson MA, et al. Urinary biomarkers for sensitive and specific detection of acute kidney injury in humans. Clin Transl Sci. 2008;1:200-8.

29. Haase-Fielitz A, Haase M, Devarajan P. Neutrophil gelatinase-associated lipocalin as a biomarker of acute kidney injury: a critical evaluation of current status. Ann Clin Biochem. 2014;51:335-51.

30. Charlton JR, Portilla D, Okusa MD. A basic science view of acute kidney injury biomarkers. Nephrol Dial Transplant Off Publ Eur Dial Transpl Assoc Eur Ren Assoc. 2014;29:1301-11.

31. Pfortmueller CA, Funk G-C, Reiterer C, et al. Normal saline versus a balanced crystalloid for goal-directed perioperative fluid therapy in major abdominal surgery: a double-blind randomised controlled study. Br J Anaesth. 2018; 120:274-83.

32. Brown RM, Wang L, Coston TD, et al. Balanced crystalloids versus saline in Sepsis. A secondary analysis of the SMART clinical trial. Am J Respir Crit Care Med. 2019:200:1487-95.

\section{Publisher's Note}

Springer Nature remains neutral with regard to jurisdictional claims in published maps and institutional affiliations.
Ready to submit your research? Choose BMC and benefit from:

- fast, convenient online submission

- thorough peer review by experienced researchers in your field

- rapid publication on acceptance

- support for research data, including large and complex data types

- gold Open Access which fosters wider collaboration and increased citations

- maximum visibility for your research: over $100 \mathrm{M}$ website views per year

At BMC, research is always in progress.

Learn more biomedcentral.com/submissions 